\title{
Avaliação Psicométrica do Questionário de Atitudes Socioculturais em Relação à Aparência-3 (SATAQ-3) para Adolescentes
}

\author{
Ana Carolina Soares Amaral ${ }^{1}$ \\ Instituto Federal de Educação, Ciência e Tecnologia do Sudeste de Minas Gerais \\ Maria Aparecida Conti \\ Universidade Cruzeiro do Sul \\ Maria Elisa Caputo Ferreira \\ Juliana Fernandes Filgueiras Meireles \\ Universidade Federal de Juiz de Fora
}

\begin{abstract}
RESUMO - Objetivou-se avaliar as qualidades psicométricas do Questionário de Atitudes Socioculturais em Relação à Aparência-3 para adolescentes brasileiros. A amostra total foi composta por 600 adolescentes (254 meninos), os quais participaram em fases distintas da pesquisa: avaliação da compreensão verbal da escala, avaliação da validade de construto, validade e confiabilidade, e a terceira etapa referiu-se à avaliação da reprodutibilidade (teste-reteste). Todos os itens apresentaram adequada compreensão verbal entre os adolescentes. O instrumento apresentou estrutura fatorial composta por sete fatores $(\alpha$ $>0,40)$, cujos índices de ajustamento foram aceitáveis, correlação positiva e significativa com a satisfação corporal $(r=0,41$, $p<0,001)$ e estabilidade. As qualidades psicométricas do SATAQ-3 para adolescentes foram parcialmente confirmadas, sendo sua utilização indicada para essa população.
\end{abstract}

Palavras-chave: psicometria, imagem corporal, adolescentes, transtornos alimentares, validade do teste

\section{Psychometric Evaluation of the Sociocultural Attitudes Towards Appearance Questionnaire-3 (SATAQ-3) among Adolescents}

\begin{abstract}
The objective of this study was to obtain estimates of the psychometric properties of the Sociocultural Attitudes Towards Appearance Questionnaire-3 in a sample of Brazilian adolescents. The sample consisted of 600 adolescents (254 boys), who participated in different stages of the research: assessment of verbal comprehension, construct validity, convergent validity and reliability, and evaluation of the reproducibility (test-retest). The adolescents showed adequate verbal comprehension of all items. The scale presented a factor structure composed of seven factors $(\alpha>0,40)$, with acceptable values of the fit indices of the model, significant positive correlation for the measures of body satisfaction $(r=0,41, p<0,001)$, and reproducibility. The psychometric quality of the SATAQ-3 among adolescents was partially confirmed, and its use is indicated for this population.
\end{abstract}

Keywords: psychometrics, body image, adolescents, eating disorders, test validity

Uma das teorias mais difundidas na atualidade acerca do desenvolvimento e manutenção da insatisfação corporal se baseia na concepção de que a exposição de ideais de beleza leva homens e mulheres a se sentirem desconfortáveis com seus corpos (Dittmar, 2005; Grabe, Ward, \& Hyde, 2008).

$\mathrm{O}$ modelo mais aceito para explicação de como ocorre essa influência é o Modelo dos Três Fatores (Amaral et al., 2013; Thompson, Coovert, \& Stormer, 1999), segundo o qual existem três fontes primárias (amigos, pais e mídia) que exercem influência no processo de organização da imagem corporal, por meio de dois mecanismos principais: a comparação da aparência e a internalização do ideal de magreza/muscularidade. A atuação destes mediadores irá determinar a presença ou não de insatisfação corporal e, consequentemente, de comportamentos alimentares inadequados (Thompson et al., 1999).

Pesquisadores têm destacado a mídia como um dos principais fatores que exerce maior influêncianesse processo

1 Endereço para correspondência: Instituto Federal de Educação, Ciência e Tecnologia do Sudeste de Minas Gerais,Campus Barbacena, Rua Monsenhor José Augusto, no 204, Bairro São José, Barbacena, MG, Brasil. CEP: 36205-018. E-mail: ana.amaral@ifsudestemg.edu.br
(Barlett, Vowels, \& Saucier, 2008; Groesz, Levine, \& Murnen, 2002; Hausenblas et al., 2013). Um dos aspectos que se relaciona à magnitude dessa influência é o tempo de exposição à mídia. Estudos apontam que até mesmo um curto tempo de contato com os modelos de corpo ideal apresentados nas revistas, na televisão, nos filmes e até mesmo nas propagandas tem papel relevante na autoestima e na satisfação corporal (Hausenblas et al., 2013). Neste sentido, Cusumano e Thompson (1997) e Tiggemann (2011) destacam os efeitos da exposição a imagens midiáticas na adoção de comportamentos de restrição alimentar e na dedicação a programas de exercícios físicos.

A avaliação desse construto em pesquisas com amostras brasileiras ainda é rara, visto a carência de instrumentos destinados a esse fim. Algumas escalas avaliam aspectos dessa influência, como os Questionários de Checagem Corporal masculino e feminino, avaliando a comparação social (Campana, Swami, Ondera, Silva, \& Tavares, 2013; Carvalho, Conti, Cordás, \& Ferreira, 2012; Kachani et al., 2013). Há, também, a Escala de Influência dos Três Fatores (Amaral et al., 2013), que avalia a influência de pais, amigos 
e mídia sobre a imagem corporal, validada para a população adulta jovem no Brasil.

Outra escala disponível para avaliação deste construto é o Questionário de Atitudes Socioculturais em Relação à Aparência (SATAQ-3) (Amaral, Cordás, Conti, \& Ferreira, 2011; Amaral, Ribeiro, Conti, Ferreira, \& Ferreira, 2013). Esse instrumento, desenvolvido originalmente por Thompson, van den Berg, Roehrig, Guarda e Heinberg (2004) é o questionário mais utilizado atualmente para avaliação da pressão sociocultural e internalização do padrão de beleza (Túry, Gülec, \& Kohls, 2010), constituindo o padrãoouro para a avaliação desse construto (López-Guimerà \& Sánchez-Carracedo, 2010; Sánchez-Carracedo et al., 2012). É composto por 30 itens divididos em quatro subescalas: internalização geral, composta por noveitens (alpha de Cronbach $=0,96$ ), que se destina a avaliar a influência geral da mídia através da televisão, das revistas e do cinema; internalização atlética, com cincoitens (alpha de Cronbach $=0,95)$, que avalia a influência dos modelos atléticos e ligados ao esporte; a terceira possuinoveitens que refletem a mídia como fonte de Informações sobre a aparência(alpha de Cronbach $=0,96)$; e, finalmente, a subescalapressão possui seteitens (alpha de Cronbach $=0,92$ ) que avaliam o sentimento pessoal a respeito da pressão exercida pelas mensagens midiáticas em relação ao corpo (Thompson et al., 2004). A consistência interna de toda a escala foi de 0,96 para a amostra de universitárias americanas.

O SATAQ-3 já teve suas qualidades psicométricas verificadas em diversas populações (Calogero, Davis, \& Thompson, 2004; Jackson \& Chen, 2010; Knauss, Paxton, \& Alsaker, 2009; Madanat, Hawks, \& Brown, 2006; Markland \& Oliver, 2008; Rousseau, Valls, \& Chabrol, 2010; Stefanile, Matera, Nerini, \& Pisani, 2011; Swami, 2009). A equivalência semântica deste instrumento para a língua portuguesa já foi avaliada (Amaral et al., 2011) e sua adaptação transcultural realizada para jovens universitários de ambos os sexos (Amaral et al., 2013) e adultos (Swami et al., 2011). Entretanto, não há registros de suas qualidades psicométricas para adolescentes brasileiros.

Segundo Thompson (2004), além da preocupação com a comprovação de validade e confiabilidade do instrumento, é necessário que o mesmo seja adequado para a amostra a qual se deseja investigar. Assim, ao considerar a adolescência como uma das etapas da vida na qual o indivíduo encontra-se mais sujeito às influências socioculturais sobre a aparência (Wertheim \& Paxton, 2011), considera-se essencial a avaliação das qualidades psicométricas desse instrumento, em especial para esta população, a fim de garantir sua aplicabilidade entre adolescentes mais jovens, os quais não foram contemplados na validação realizada por Amaral et al. (2011).

Desta forma, o presente estudo buscou avaliar a compreensão verbal, estrutura fatorial, validade convergente, consistência interna e reprodutibilidade do SATAQ-3 entre adolescentes brasileiros.

\section{Método}

\section{Etapas e Participantes}

Participaram deste estudo adolescentes estudantes de escolas públicas da cidade de Juiz de Fora/Minas Gerais, selecionados por conveniência, de acordo com a disponibilidade em participar do estudo e presença em sala de aula nos dias de aplicação dos questionários.

$\mathrm{O}$ estudo foi realizado em três etapas. Na primeira, avaliou-se a compreensão verbal da versão do instrumento traduzido para a língua portuguesa (Amaral et al., 2011) pelos adolescentes. A amostra para esta etapa foi composta por 71 indivíduos, 49 meninas e 22 meninos, com média de idade de 13,1 anos $(D P=0,7)$ e 13,5 anos $(D P=1,0)$, respectivamente.

A segunda etapa referiu-se à avaliação da estrutura fatorial do instrumento, da qual participaram 529 adolescentes (297 meninas e 232 meninos). A idade variou de 13 a 23 anos, com média de 15,9 anos $(D P=1,7)$ entre as meninas e 15,9 anos $(D P=1,6)$ entre os meninos. O Índice de Massa Corporal médio derivado do peso e estatura auto referidos foi de $21,5(D P=3,8)$ e $21,4(D P=3,7)$ para meninas e meninos, respectivamente.

Para a terceira etapa, foram selecionadas aleatoriamente, do total da amostra, três turmas para a avaliação da reprodutibilidade. A amostra para esta fase foi de 66 adolescentes, sendo 42 meninas, com média de idade de 16,9 anos $(D P=1,5)$ e 24 meninos, com média de idade de $17,3 \operatorname{anos}(D P=1,3)$.

\section{Instrumentos}

Questionário de Atitudes Socioculturais em Relação à Aparência (SATAQ-3). O SATAQ-3 é composto por 30 questões, com respostas na forma de escala Likert de1 (discordo totalmente) a 5 (concordo totalmente), destinadas a avaliar a influência da mídia em relação ao corpo. $O$ escore final é calculado pela soma das respostas, e a pontuação representa proporcionalmente a influência dos aspectos socioculturais na imagem corporal. Os itens do questionário englobam quatro formas de influência: a) internalização geral dos padrões socialmente estabelecidos (questões 3, 4, 7, 8, $11,12,15,16,27$ ); b) ideal de corpo atlético (questões 19, 20, 23, 24, 30); c) pressão exercida por esses padrões sobre a imagem corporal (questões 2, 6, 10, 14, 18, 22, 26); e d) mídia como fonte de informações sobre aparência (questões $1,5,9,13,17,21,25,28,29)$. A versão utilizada neste estudo foi proposta por Amaral et al. (2011), que avaliaram a equivalência semântica do instrumento para o idioma português.

Body Shape Questionnaire (BSQ). Esse instrumento possui 34 perguntas em escala Likert de pontos, variando de 1 (nunca) a 6 (sempre), desenvolvido por Cooper, Taylor, Cooper e Fairburn (1987) para avaliar a preocupação com a forma e peso corporais. A versão utilizada neste estudo foi validada para adolescentes brasileiros, de ambos os sexos, por Conti, Cordás e Latorre (2009), obtendo adequados índices de validade e confiabilidade (alpha de Cronbach $=0,96$; 
coeficiente de correlação intra-classe $=0,91 ; p<0,001)$. A pontuação varia de 34 a 204 pontos, sendo que quanto maior o escore obtido, maior a insatisfação corporal. Para a amostra deste estudo, este instrumento obteve consistência interna de 0,95 para meninos e 0,96 para meninas. O BSQ foi incluído neste estudo a fim de avaliar a validade convergente do SATAQ-3, visto a comprovada associação entre a influência da mídia e a insatisfação corporal (Amaral et al., 2013).

\section{Procedimentos}

Em um primeiro momento, os responsáveis pelas instituições de ensino onde seriam realizadas as coletas foram contatados e autorizaram a realização da pesquisa. Após a autorização da direção das escolas, os Termos de Consentimento Livre e Esclarecido (TCLE) foram entregues aos alunos, que foram informados sobre todos os procedimentos necessários para a realização da pesquisa. Apenas participaram do estudo os adolescentes que apresentaram o TCLE assinado pelo responsável.

Para avaliação da compreensão verbal dos itens do SATAQ-3 pelos adolescentes, o instrumento foi aplicado na forma de uma escala verbal numérica adaptada (Amaral et al., 2011; Conti, Scagliusi, Queiroz, Hearst, \& Cordás, 2010), na qual os participantes deveriam informar em uma escala Likert o quanto compreenderam do enunciado $(0=$ não entendi nada a 5 = entendi tudo e não tenho dúvidas). Os participantes podiam, ainda, fornecer sugestões para que o enunciado fosse mais bementendido. Este procedimento teve como objetivo verificar se as questões traduzidas para o idioma português (Brasil) (Amaral et al., 2011) eram de fácil compreensão para os adolescentes.

Para a segunda etapa, a aplicação dos questionários foi conduzida em grupos, em sala de aula, entre os meses de novembro e dezembro de 2012. Os alunos responderam individualmente aos questionários e a um cabeçalho de identificação. As coletas foram realizadas por um mesmo pesquisador, que padronizou as orientações verbais. Os participantes do estudo não se comunicaram entre si e não houve limite de tempo para preenchimento dos instrumentos.

Foram selecionadas, aleatoriamente, três turmas participantes da segunda etapa a fim de avaliar-se a reprodutibilidade. Para tanto, realizou-se a aplicação do instrumento em dois pontos no tempo, com intervalo de duas semanas entre as aplicações.

O projeto do presente estudo foi aprovado pelo Comitê de Ética e Pesquisa da Universidade Federal de Juiz de Fora (UFJF), em 15/07/10 (Parecer n ${ }^{\circ}$. 148/2010), o qual trata da avaliação das propriedades psicométricas do SATAQ-3 em duas amostras distintas: jovens universitários e adolescentes. Sua execução está de acordo com as normas da Portaria 466/12 do Conselho Nacional de Saúde (Brasil).

\section{Análise Estatística}

Os valores obtidos para a compreensão verbal foram analisados de forma descritiva, através de média e desvios padrão. Considerou-se de adequada compreensão os itens que obtiveram escore médio superior a 4 (quatro) (Amaral et al., 2011; Conti et al., 2010).

A estrutura fatorial do instrumento foi avaliada através de Análise Fatorial Exploratória (AFE). Em um primeiro momento, foi realizada uma análise de componentes principais categóricos (CatPCA), utilizando-se o número de dimensões igual ao número de itens, com o intuito de transformar os dados ordinais derivados da escala Likert em variáveis numéricas. Apenas após este processo é que os dados foram submetidos à AFE, utilizando como método de extração a Análise de Componentes Principais e rotação varimax, a qual é um método de rotação ortogonal que minimiza o número de variáveis com cargas altas em cada fator, simplificando, assim, a interpretação dos fatores (Lattin, Carroll, \& Green, 2011). Como critério de seleção dos fatores adotou-se autovalor maior que 1 e cargas significativas aquelas superiores a 0,4 (Hair Junior, Anderson, Tatham, \& Black, 2005).

Realizou-se, também, análise fatorial confirmatória (AFC) com o intuito de validar a estrutura fatorial obtida na AFE. Como os dados oriundos da CatPCA possuem distribuição normal, utilizou-se o método de Máxima Verossimilhança e o ajustamento do modelo foi avaliado através dos seguintes índices: Comparative Fit Index (CFI), Normed Fit Index (NFI) e Tucker-Lewis Index (TLI), para os quais valores acima de 0,80 são aceitáveis, sendo considerados ótimos os valores acima de 0,95 (Brown, 2006; Kahn, 2006); e o Root Mean Square Error of Approximation (RMSEA), para o qual são esperados valores inferiores a 0,08 (Pilati \& Laros, 2007), sendo considerado um bom ajustamento do modelo valores menores que 0,06 (Hu \&Bentler, 1999; Kahn, 2006).

Para avaliação da validade convergente, os escores obtidos pelos dois instrumentos (SATAQ-3 e BSQ) foram inicialmente convertidos em escore $\mathrm{z}$, a fim de padronizálos. Por representarem variáveis ordinais (escala Likert), optou-se pela utilização do teste de correlação de Spearman. Calculou-se a consistência interna através do coeficiente alpha de Cronbach. A reprodutibilidade foi avaliada através do teste de Wilcoxon-Mann-Whitney, no qual as médias dos escores obtidos pelos participantes nos momentos 1 e 2 foram comparadas, e pelo cálculo do coeficiente de correlação intra-classe.

Para as análises de estatística descritiva, inferencial e para a AFE, utilizou-se o software Statistical Package for the Social Sciences v.21.0. A Análise Fatorial Confirmatória foi realizada utilizando-se o software Amos v.21.0.

\section{Resultados}

\section{Compreensão Verbal}

Todos os itens obtiveram escores de compreensão verbal superiores a 4. A menor média foi para o item 9, o qual apresentou escore médio de 4,0 $(D P=1,6)$. O item foi revisto por um comitê de especialistas, o qual optou pela manutenção da mesma forma semântica, visto não ter havido nenhuma sugestão por parte dos adolescentes para a reestruturação da 
questão e esta ter atendido aos requisitos de compreensão verbal pré-estabelecidos.

\section{Análise Fatorial Exploratória}

A medida de adequação da amostra Kaiser-Meyer-Olkin (KMO) foi de 0,86 e o teste de esfericidade de Bartlett teve valor $\chi^{2}=3173(p<0,001)$, indicando que a amostra apresentou-se adequada à AFE.

Tabela 1. Cargas Fatoriais com Rotação Varimax do SATAQ-3 para adolescentes ${ }^{1}$. Juiz de Fora, 2013

\begin{tabular}{|c|c|c|c|c|c|c|c|}
\hline \multirow[t]{2}{*}{ Itens } & \multicolumn{7}{|c|}{ Fatores } \\
\hline & 1 & 2 & 3 & 4 & 5 & 6 & 7 \\
\hline 4. Comparo meu corpo com os de pessoas que estão na TV. & 0,482 & & & & & & \\
\hline 7. Gostaria que meu corpo fosse parecido com os do(as) modelos das revistas. & 0,743 & & & & & & \\
\hline 11. Gostaria que meu corpo fosse parecido com os dos(as) modelos dos filmes. & 0,696 & & & & & & \\
\hline 15. Gostaria de me parecer com os(as) modelos de videoclipes. & 0,708 & & & & & & \\
\hline 16. Comparo minha aparência com a das pessoas das revistas. & 0,500 & & & & & & \\
\hline 20. Comparo meu corpo ao das pessoas em boa forma. & 0,415 & & & & & & \\
\hline 2. Já me senti pressionado(a) pela TV ou por revistas a perder peso. & & 0,679 & & & & & \\
\hline 10. Já me senti pressionado(a) pela TV ou pelas revistas a ser magra/musculoso. & 0,475 & 0,473 & & & & & \\
\hline 14. Já me senti pressionado(a) pela TV ou pelas revistas a ter um corpo perfeito. & 0,421 & $\mathbf{0 , 5 3 3}$ & & & & & \\
\hline 18. Já me senti pressionado(a) pela TV ou por revistas a fazer dieta. & & 0,704 & & & & & \\
\hline 22. Já me senti pressionado(a) pela TV ou pelas revistas a praticar exercícios. & & 0,580 & & & & & \\
\hline 26. Já me senti pressionado(a) pela TV ou pelas revistas a mudar minha aparência. & & 0,626 & & & & & \\
\hline $\begin{array}{l}\text { 17. Anúncios em revistas são importantes fontes de informação sobre moda e sobre } \\
\text { "como ser atraente". }\end{array}$ & & & 0,473 & & & & \\
\hline $\begin{array}{l}\text { 21. Fotos de revistas são importantes fontes de informação sobre moda e sobre "como } \\
\text { ser atraente". }\end{array}$ & & & 0,650 & & & & \\
\hline $\begin{array}{l}\text { 25. Filmes são importantes fontes de informação sobre moda e sobre "como ser } \\
\text { atraente". }\end{array}$ & & & 0,641 & & & & \\
\hline $\begin{array}{l}\text { 29. Pessoas famosas são importantes fontes de informação sobre moda e sobre "como } \\
\text { ser atraente". }\end{array}$ & & & 0,719 & & & & \\
\hline 23. Gostaria de ter uma aparência tão atlética quanto a das estrelas do esporte. & & & & 0,763 & & & \\
\hline 24. Comparo meu corpo com o de pessoas atléticas. & & & & 0,639 & & & \\
\hline 30. Tento me parecer com atletas. & & & & 0,761 & & & \\
\hline 6. Não me sinto pressionado(a) pela TV ou pelas revistas a ficar bonita/musculoso. & & & & & 0,444 & & \\
\hline $\begin{array}{l}\text { 9. Videoclipes não são importantes fontes de informação sobre moda e sobre "como ser } \\
\text { atraente". }\end{array}$ & & & & & 0,661 & & \\
\hline 12. Não comparo meu corpo com os das pessoas das revistas. & & & & & 0,537 & & \\
\hline $\begin{array}{l}\text { 13. Artigos de revistas não são importantes fontes de informação sobre moda e sobre } \\
\text { "como ser atraente". }\end{array}$ & & & & & 0,456 & & \\
\hline $\begin{array}{l}\text { 28. Estrelas de cinema não são importantes fontes de informação sobre moda e sobre } \\
\text { "como ser atraente". }\end{array}$ & & & & & 0,533 & & \\
\hline $\begin{array}{l}\text { 1. Programas de TV são importantes fontes de informação sobre moda e sobre "como } \\
\text { ser atraente". }\end{array}$ & & & & & & 0,709 & \\
\hline $\begin{array}{l}\text { 5. Comerciais de TV são importantes fontes de informação sobre moda e sobre "como } \\
\text { ser atraente". }\end{array}$ & & & & & & 0,746 & \\
\hline 3. Não me importo se meu corpo se parece com os de pessoas que estão na TV & & & & & & & 0,539 \\
\hline 8. Comparo minha aparência com a das estrelas de TV e do cinema. & & & & & & & 0,573 \\
\hline 19. Não desejo ser tão atlético(a) quanto as pessoas das revistas. & & & & & & & 0,423 \\
\hline 27. Não tento me parecer com as pessoas da TV. & & & & & & & 0,559 \\
\hline Autovalor & 3,194 & 2,710 & 2,466 & 2,209 & 1,838 & 1,771 & 1,515 \\
\hline \% variância explicada & 10,6 & 9,03 & 8,22 & 7,36 & 6,13 & 5,90 & 5,05 \\
\hline Alfa de Cronbach & 0,81 & 0,76 & 0,67 & 0,72 & 0,51 & 0,63 & 0,40 \\
\hline
\end{tabular}

Nota. ${ }^{1}$ As cargas inferiores a 0,40 foram suprimidas da tabela 
A solução fatorial oriunda da AFE apresentou setefatores que explicaram $52,34 \%$ da variância total do instrumento. A título de exploração dos dados, forçou-se uma solução fatorial com quatro fatores, como no instrumento original. A estrutura fatorial derivada desta análise apresentou variância explicada de 39,96\% e vários itens não apresentaram carga fatorial significativa em nenhum dos fatores. Assim, optou-se por adotar como estrutura fatorial mais adequada aquela com setefatores, de acordo com a Tabela 1. Os números em negrito correspondem aos itens que apresentaram carga superior a 0,4 em mais de um fator.

O fator 1 agrupou itens da dimensão internalização geral acrescido do item 20, originalmente derivado da subescalainternalização atlética. O fator 2 foi representado apenas por itens da subescalapressão, sendo que os itens 10 e 14, designados originalmente nesta última, apresentaram carga fatorial dividida entre os fatores 1 e 2. O fator 3 aglutinou os itens da subescalainformação referentes a fontes como revistas e filmes, e o fator 6 representou a dimensão informação oriunda da televisão. Já o fator 4 agrupou itens da subescalainternalização atlética e no fator 5 carregaram fortemente os itens da escala que apresentam direção negativa, representando o fator Escore Reverso. O fator 7 agrupou itens da dimensão internalização geral, acrescido do item 19, originalmente da subescalainternalização atlética.

Assim, a estrutura fatorial do SATAQ-3 para adolescentes brasileiros obteve setefatores, sendo o fator 1 composto por seis itens relacionados à internalização geral, o fator 2 com seisitens destinados à avaliação da pressão, o fator 3 com quatroitens referentes à informação oriunda de fontes diversas, o fator 4 apresentou trêsitens relacionados à internalização atlética, o fator 5 com cincoitens avaliando todas as dimensões mas com escore reverso, o fator 6 aglutinou os doisitens relacionados à informações advindas da televisão e, por fim, o fator 7 foi composto por quatroitens relacionados, também, à internalização geral.

\section{Análise Fatorial Confirmatória}

AAFC foi realizada considerando-se os fatores derivados da AFE como construtos exógenos. Os valores obtidos para os índices de ajustamento foram: $C F I=0,86 ; N F I=0,77$; $T L I=0,84$ e $R M S E A=0,046$. Segundo esses índices, podese considerar que o ajustamento do modelo não foi o mais adequado, entretanto, apresentou valores aceitáveis (Kahn, 2006; Thompson, 2004).

Com base nesses resultados, testou-se o ajustamento do modelo de quatrofatores da escala original, assim como o dos cinco fatores derivado da validação para adultos jovens brasileiros. Entretanto, nenhum desses modelos alternativos apresentou melhora nos índices de ajustamento, apresentando, pelo contrário, valores inferiores aos encontrados no modelo com setefatores.

A matriz de correlação entre os fatores está apresentada na Tabela 2. Todas as correlações foram significativas ( $p$ $<0,001)$, indicando que os construtos exógenos não são independentes entre si.

\section{Validade Convergente}

Os valores da correlação entre os escores do SATAQ-3 e do BSQ estão apresentados na Tabela 3 e indicam que quanto maior a insatisfação corporal medida pelo BSQ, maior também a influência sociocultural de acordo com o SATAQ-3, tanto para a amostra total quanto para os grupos estratificados por sexo.

\section{Consistência Interna}

Foi encontrada adequada consistência interna tanto para a amostra total $(\alpha=0,84)$ quanto para meninos e meninas, com

Tabela 2. Matriz de Correlação das Variáveis Latentes para Análise Fatorial Confirmatória do SATAQ-3. Juiz de Fora, 2013

\begin{tabular}{|c|c|c|c|c|c|c|}
\hline & 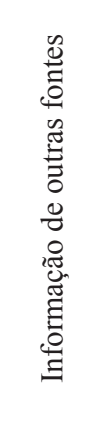 & 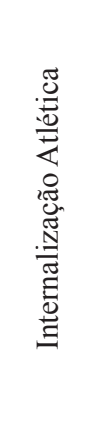 & 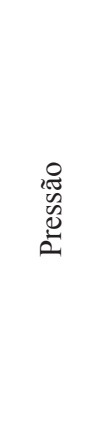 & 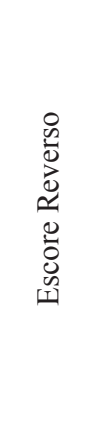 & 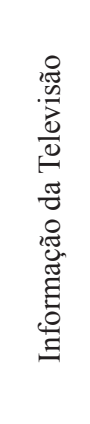 & 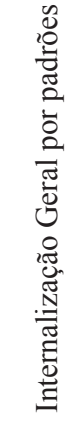 \\
\hline Internalização Geral por comparação & 0,503 & 0,587 & 0,751 & 0,424 & 0,321 & 0,493 \\
\hline Informação de outras fontes & & 0,418 & 0,518 & 0,354 & 0,653 & 0,040 \\
\hline Internalização Atlética & & & 0,494 & 0,136 & 0,177 & 0,420 \\
\hline Pressão & & & & 0,442 & 0,330 & 0,391 \\
\hline Escore Reverso & & & & & 0,257 & 0,760 \\
\hline Informação da Televisão & & & & & & 0,420 \\
\hline
\end{tabular}


valores de alpha de Cronbach de 0,83 e 0,86 respectivamente. Os valores de consistência interna para cada um dos fatores encontrados estão apresentados na Tabela 1 .

\section{Reprodutibilidade}

As análises não revelaram diferença significativa entre os escores obtidos nos dois momentos, seja para todos os participantes ou para a amostra estratificada segundo o sexo. Os valores de $p$ encontram-se na Tabela 3, assim como os valores dos coeficientes de correlação intra-classe.

\section{Discussão}

As qualidades psicométricas do SATAQ-3 entre os adolescentes foram parcialmente confirmadas através da comprovação de sua estrutura fatorial, apresentado valores adequados de consistência interna e reprodutibilidade, bem como associação significativa com a insatisfação corporal. Apesar de os valores de ajustamento do modelo fatorial não serem considerados ótimos, Herdman, Fox-Rushby e Badia (1998) destacam que os resultados da análise de fatores não devem ser considerados de forma isolada, mas juntamente com outras medidas de validade e confiabilidade do instrumento.

Em relação à compreensão verbal, pode-se considerar que todos os itens do SATAQ-3 apresentaram compreensão satisfatória, podendo este, portanto, ser utilizado em amostras de adolescentes sem necessidade de outras adaptações. Destaca-se que, no estudo de avaliação semântica deste instrumento, a compreensão de seus itens já havia sido atestada entre adultos universitários brasileiros (Amaral et al., 2011).

Para a análise fatorial, apenas os itens 10 e 14 apresentaram cargas semelhantes entre dois fatores. Por representarem, originalmente, avaliação da dimensão pressão, optou-se pela manutenção destes itens no fator 2. Assim, a estrutura fatorial apresentou setefatores, com as dimensões refletindo internalização geral por comparação (Fator 1), pressão (Fator 2), informações advindas da televisão (Fator 6), informações advindas de outras fontes midiáticas (Fator 3), internalização atlética (Fator 4), internalização geral por padrões (Fator 7) e questões de escore reverso (Fator 5).

Segundo Sánchez-Carracedo et al. (2012), a estrutura fatorial original do SATAQ-3 não tem sido replicada em suas diferentes versões e adaptações. Como exemplos, pode-se citar a versão para adolescentes chineses do sexo masculino, a qual apresentou quatrofatores cujos itens aglutinados não refletiram nenhuma das dimensões originais (Jackson \& Chen, 2010) e a versão para mulheres malaias (Swami, 2009), na qual dois dos quatro fatores obtidos representaram a aglutinação de itens de dois ou mais fatores. Entretanto, essas diferenças não invalidam a estrutura do instrumento. Apenas indicam interpretações diferentes dos itens em cada uma das culturas nas quais é utilizado.

Outro item que apresentou carga significativa em um fator diferente de sua dimensão original foi a questão 20 (“Comparo meu corpo ao das pessoas em boa forma"). Este, originalmente da subescala internalização atlética, apresentou carga fatorial mais elevada no fator 1 , de internalização geral. Outros estudos também verificaram comportamento semelhante desse item (Amaral et al., 2013; Calogero et al., 2004; Markland \& Oliver, 2008). Sánchez-Carracedo et al. (2012) destacam que uma possível justificativa para essa aglutinação é que os demais itens da subescala internalização atlética se referem a esportistas ou atletas como fontes de comparação, enquanto o item 20 diz respeito a pessoas em "boa forma". Esses autores chegam, inclusive, a recomendar a exclusão deste item da escala. Entretanto, não se considera que os resultados deste estudo reafirmem esta necessidade, visto que apesar de ter carregado em um fator diferente do seu original, o item 20 apresentou carga significativa $(>0,40)$ apenas em um fator.

No que diz respeito à $\mathrm{AFC}$, os índices de ajustamento foram próximos aos valores de referência. Hair Junior, Black, Babin, Anderson e Thatam (2009) destacam que em modelos mais complexos e com amostras maiores, como é o caso do presente estudo, valores de corte de 0,95 para estas medidas de ajustamento são irreais. Sendo assim, pode-se dizer que o modelo foi aceitável, visto os índices terem se aproximado de 0,80 ou mesmo superaram esse valor. Em relação ao $R M S E A$, medida mais importante no que diz respeito à extrapolação do modelo para a população e não apenas para a amostra (Thompson, 2004), pode-se dizer que os valores foram satisfatórios, pois foram inferiores ao índice de referência de 0,06 $(R M S E A=0,046)$. Assim, considera-se que o modelo fatorial foi validado através da AFC, podendo ser extrapolado para amostras semelhantes à deste estudo.

Os valores encontrados para a correlação do SATAQ-3 ao BSQ indicaram boa validade convergente do instrumento, comprovando sua associação à insatisfação corporal. No estudo de desenvolvimento do SATAQ-3 (Thompson et al., 2004), os autores já apontavam para a associação entre a influência sociocultural e a insatisfação corporal, fato comprovado através destes resultados. Resultado semelhante também foi encontrado na adaptação transcultural deste instrumento para universitários brasileiros (Amaral et al., 2013), indicando a existência desta relação também em amostras brasileiras.

A consistência interna do instrumento mostrou-se adequada tanto para a amostra total quanto para os grupos estratificados de acordo com o sexo, obtendo valores superiores a 0,80. Entretanto, ao analisar-se este parâmetro para cada um dos setefatores, as dimensões 5 e 7 apresentaram valores inferiores a 0,60 , considerados valores de alpha de Cronbach insuficientes. $\mathrm{O}$ fator 5 representa uma miscelânea de itens oriundos de fatores diversos, todos de direção negativa. Alguns autores têm apontado os itens negativos do SATAQ-3 como problemáticos entre algumas amostras, apresentando valores de validade e confiabilidade menores que o restante dos itens (Amaral et al., 2011; Markland\& Oliver, 2008; Wheeler, Vassar, \& Hale, 2011). Já o fator 7, cujo valor alpha pode ser classificado como inaceitável, apresentou itens que avaliam a internalização, porém em relação a diferentes influências (geral e atlética). Talvez, esta diferença em relação ao tipo de internalização pode ter influenciado para a não homogeneidade dos itens. Segundo Raykov e Marcoulides (2011), o alpha de Cronbach não será 
Tabela 3. Valores Referentes à Validade Convergente, Confiabilidade e Reprodutibilidade do SATAQ-3 entre Adolescentes. Juiz de Fora, 2013

\begin{tabular}{|c|c|c|c|c|c|c|c|c|}
\hline Análise & Variável & Parâmetro & & & Participan & & & \\
\hline \multirow{2}{*}{$\begin{array}{l}\text { Validade } \\
\text { Convergente }\end{array}$} & & & $\begin{array}{l}\text { Sexo masculino } \\
(\mathrm{n}=232)\end{array}$ & $p$ & $\begin{array}{l}\text { Sexo feminino } \\
(\mathrm{n}=297)\end{array}$ & $p$ & $\begin{array}{l}\text { Total } \\
(\mathrm{n}=529)\end{array}$ & $\mathrm{p}$ \\
\hline & $\begin{array}{l}\text { SATAQ-3 - } \\
\text { BSQ }\end{array}$ & Rho & 0,33 & $<0,001$ & 0,47 & $<0,001$ & 0,41 & $<0,001$ \\
\hline Confiabilidade & $\begin{array}{l}\text { Consistência } \\
\text { Interna }\end{array}$ & $\begin{array}{l}\text { Alfa de } \\
\text { Cronbach }\end{array}$ & 0,83 & & 0,86 & & 0,84 & \\
\hline \multirow[t]{4}{*}{ Reprodutibilidade } & & & $\mathrm{n}=24$ & & $\mathrm{n}=42$ & & $\mathrm{n}=66$ & \\
\hline & $\begin{array}{l}\text { Escores do } \\
\text { SATAQ-3 }\end{array}$ & $\begin{array}{l}\text { Média T1 } \\
\text { (DP) }\end{array}$ & $70,32(24,21)$ & 0,46 & $79,21(23,01)$ & 0,71 & $\begin{array}{l}76,02 \\
(23,61)\end{array}$ & 0,52 \\
\hline & & $\begin{array}{l}\text { Média T2 } \\
\text { (DP) }\end{array}$ & $69,30(22,36)$ & & $77,84(21,91)$ & & $\begin{array}{l}74,84 \\
(22,25)\end{array}$ & \\
\hline & $\mathrm{T} 1 \times \mathrm{T} 2$ & $r_{\text {icc }}$ & 0,95 & $<0,001$ & 0,78 & $<0,001$ & 0,86 & $<0,001$ \\
\hline
\end{tabular}

Nota: $\mathrm{n}$ - tamanho da amostra; T1 - escores do teste; T2 - escores do reteste (2 semanas após T1)

uma boa medida de confiabilidade a menos que todos os itens carreguem uniformemente em sua dimensão latente comum, o que não é o caso do fator 7 obtido no presente estudo. Ressalta-se, entretanto, que a consistência interna, apesar de um importante parâmetro para avaliação da confiabilidade, não deve ser avaliado isoladamente, mas em conjunto com as cargas fatoriais, por exemplo (Raykov \& Marcoulides, 2011; Sánchez-Carracedo et al., 2012).

A reprodutibilidade do instrumento foi confirmada pelos altos valores de correlação intra-classe e a não existência de diferença significativa entre os escores nos momentos 1 e 2, tanto entre a amostra total quanto para meninos e meninas. Vale destacar que esta qualidade psicométrica não tem sido avaliada nas adaptações transculturais deste instrumento. Apenas o estudo de validação do SATAQ-3 para universitários brasileiros realizou a avaliação da reprodutibilidade, obtendo resultados semelhantes aos do presente estudo, que comprovaram a estabilidade após intervalo de duassemanas (Amaral et al., 2013).

Apesar de seus avanços, este estudo apresentou limitações. Entre elas, destaca-se a utilização de medidas de peso e altura auto referidas. Entretanto, destaca-se que em função da dificuldade de realização de aferições diretas em amostras numerosas, alguns autores comprovaram que, em relação à altura e ao peso, a diferença entre a informação dada pelo participante e a medida aferida é mínima (AvilaFunes, Guitierrez-Robledo, \& Ponce de Leon Rosales, 2004; Kawada\& Suzuki, 2005), justificando sua utilização em estudos de validade. Além disso, diversos estudos que buscaram avaliar as propriedades psicométricas de instrumentos de avaliação da imagem corporal utilizaram este recurso sem prejuízo à qualidade das informações (Amaral et al., 2013; Conti et al., 2013; Scagliusi et al., 2006).

Outra importante limitação diz respeito à utilização de questionários auto aplicativos como medidas de validade convergente, podendo não refletir adequadamente os construtos investigados. Justifica-se, entretanto, que este tipo de instrumento é o mais utilizado na avaliação da imagem corporal e a escala utilizada (BSQ) já obteve comprovadas validade e confiabilidade entre adolescentes brasileiros (Conti et al., 2009).Em relação à amostra, pode-se considerar uma limitação a utilização do mesmo grupo de participantes para a avaliação da estrutura fatorial (AFE e AFC). Entretanto, diversos estudos semelhantes a este adotaram os mesmos procedimentos (Amaral et al., 2013; Bonfim, Santos, Menezes, Reichenheim, \& Barreto, 2011; Carvalho, Conti, Ribeiro, Amaral, \& Ferreira, no prelo). Apesar disso, considera-se que a realização da AFC representa um avanço, visto muitos pesquisadores optarem por não realizar esta técnica em função da necessidade de amostras numerosas (Pilati \& Laros, 2007).

Ademais, esse é o primeiro estudo que avalia uma escala específica de pressão sociocultural para adolescentes brasileiros. Mesmo não respondendo a todas as exigências estatísticas, sabe-se que esta população revela particularidades e especificidades atreladas à formação e cultura brasileiras. Ou seja, pensam, sentem e refletem de forma diferente em relação aos adolescentes de outros países, como já descritos em outros estudos (Jackson \& Chen, 2010; Swami, 2009). Sendo assim, como um estudo precursor, a presente pesquisa sinaliza para algumas diferenças que devem ser respondidas em investigações posteriores.

A partir dos resultados encontrados, conclui-se que a versão em português do SATAQ-3 obteve indícios de boas qualidades psicométricas entre adolescentes brasileiros, de ambos os sexos. Espera-se que este instrumento possa ser utilizado em investigações a respeito da influência sociocultural sobre a imagem corporal desta população, visto ser um grupo de risco para o desenvolvimento de transtornos alimentares e psicológicos. 


\section{Referências}

Amaral, A. C. S., Cordás, T. A., Conti, M. A., \& Ferreira, M. E. C. (2011). Equivalência semântica e avaliação da consistência interna da versão em português do Sociocultural AttitudesTowardsAppearance Questionnaire-3 (SATAQ-3). Cadernos de Saúde Pública, 27(8), 1487-1497.doi:10.1590/ S0102-311X2011000800004

Amaral, A. C. S., Ferreira, M. E. C., Scagliusi, F., Costa, L. S., Cordás, T. A., \& Conti, M. A. (2013). Avaliação psicométrica da Escala de Influência dos Três Fatores (EITF) de insatisfação corporal e transtorno alimentar para o idioma português. Psicologia: Reflexão e Crítica, 26(2), 213-221.doi:10.1590/ S0102-79722013000200001

Amaral, A. C. S., Ribeiro, M. S., Conti, M. A., Ferreira, C. S., \& Ferreira, M. E. C. (2013). Psychometric evaluation of the Sociocultural Attitudes Towards Appearance Questionnaire-3 among Brazilian Young adults.The Spanish Journal of Psychology, 16(e94), 1-10.doi:10.1017/sjp.2013.94

Avila-Funes, J. A., Gutierrez-Robledo, L. M., \& Ponce de Leon Rosales, S. (2004). Validity of height and weight self-report in Mexican adults: results from the national health and aging study. Journal of Nutrition, Health \& Aging, 8(5), 355-361.

Barlett, C. P., Vowels, C. L., \& Saucier, D. A. (2008). MetaAnalyses of the Effects of Media Images on Men's Body-image Concerns.Journal of Social and Clinical Psychology,27(3), 279-310.doi:10.1521/jscp.2008.27.3.279

Bonfim, C. B., Santos, D. N., Menezes, I. G., Reichenheim, M. E., \& Barreto, M. L. (2011). Um estudo sobre a validade de construto da Parent-ChildConflictTacticsScale (CTSPC) em uma amostra populacional urbana do Nordeste brasileiro. Cadernos de Saúde Pública, 27(11), 2215-2226.doi:10.1590/ S0102-311X2011001100015

Brown, T. A. (2006). Confirmatory Factor Analysis for Applied Research. New York: The Guilford Press.

Calogero, R. M., Davis, W. N., \& Thompson, J. K. (2004). The Sociocultural Attitudes Towards Appearance Quationnaire (SATAQ - 3): reliability and normative comparisons of eating disordered patients. BodyImage, 1(2), 193-198.doi:10.1016/j. bodyim.2004.01.004

Campana, A. N., Swami, V., Onodera, C. M., Silva, D., \& Tavares, M. C. G. C. F. (2013). An initial psychometric evaluation and exploratory cross-sectional study of the body checking questionnaire among Brazilian women. PLoSOne, 8(9), 1-10. doi:10.1371/journal.pone.0074649

Carvalho, P. H. B., Conti, M. A., Cordás, T. A., \& Ferreira, M. E. C. (2012). Tradução para o português (Brasil), equivalência semântica e consistência interna do Male BodyCheckingQuestionnaire (MBCQ). Revista de Psiquiatria Clínica, 39(2), 74-75. doi:10.1590/S0101-60832012000200006

Carvalho, P. H. B., Conti, M. A., Ribeiro, M. S., Amaral, A. C. S., \& Ferreira, M. E. C. (no prelo). Avaliação psicométrica do Male Body Checking Questionnaire (MBCQ). Psicologia: Reflexão e Crítica.

Conti, M. A., Cordás, T. A., \&Latorre, M. R. D. O. (2009). A study of the validity and reliability of the Brazilian version of the Body Shape Questionnaire (BSQ) among adolescents. Revista Brasileira de Saúde Materno Infantil, 9(3), 331-338. doi:10.1590/S1519-38292009000300012
Conti, M. A., Scagliusi, F. B., Queiroz, G. K. O., Hearst, N., \&Cordás, T. A. (2010). Adaptação transcultural: tradução e validação de conteúdo para o idioma português do modelo da Tripartite InfluenceScale de insatisfação corporal. Cadernos de Saúde Pública, 26(3), 503-513.doi:10.1590/S0102311X2010000300008

Conti, M. A., Ferreira, M. E. C., Carvalho, P. H. B., Kotati, M. S., Fassarella, E. S., Costa, L. S. ...Scagliusi, F. B. (2013). Stunkard figure rating scale for Brazilian men. Eating and Weight Disorder, 18(3), 317-322. doi:10.1007/s40519-0130037-8

Cooper, P. J., Taylor, M. J., Cooper, Z., \& Fairburn, C. G. (1987). The development and validation of the Body Shape Questionnaire. International Journal of Eating Disorders, 6(4), 485-494.doi:10.1002/1098-108X(198707)6:4<485::AIDEAT2260060405>3.0.CO;2-O

Cusumano, D. L., \& Thompson, J. K. (1997). Body image and body shape ideals in magazines: exposure, awareness, and internalization. Sex Roles, 37(9-10), 701-721.doi:10.1007/ BF02936336

Dittmar, H. (2005). Vulnerability factors and processes linking sociocultural pressures and body dissatisfaction. Journal of Social and Clinical Psychology, 24(8), 1081-1087.doi:10.1521/ jscp.2005.24.8.1081

Grabe, S., Ward, L. M., \& Hyde, J. S. (2008). The role of the media in body image concerns among women: a meta-analysis of experimental and correlational studies. Psychological Bulletin, 134(3), 460-476.doi:10.1037/0033-2909.134.3.460

Groesz, L. M., Levine, M. P., \&Murnen, S. K. (2002). The effect of experimental presentation of thin media images on body satisfaction: A meta-analytic review. International Journal of Eating Disorders, 31(1), 1-16. doi:10.1002/eat.10005

Hair Junior., J. F., Anderson, R. E., Tatham, R. L., \& Black, W. C. (2005). Análise Multivariada de dados (5a ed.). Porto Alegre: Bookman.

Hair Junior, J. F., Black, W. C., Babin, B. J., Anderson, R. E., \& Tatham, R. L. (2009). Análise Multivariada de dados (6a ed.). Porto Alegre: Bookman.

Hausenblas, H. A., Campbell, A., Menzel, J. E., Doughty, J., Levine, M., \& Thompson, J. K. (2013). Media effects of experimental presentation of the ideal physique on eating disorder symptoms: A meta-analysis of laboratory studies. Clinical Psychological Review,33(1), 168-181.doi:10.1016/j.cpr.2012.10.011

Herdman, M., Fox-Rushby, J., \& Badia, X. (1998). A model of equivalence in the cultural adaptation of HRQoL instruments: the universalist approach. Quality of Life Research, 7(4), 32335.doi:10.1023/A:1024985930536

Hu, L., \& Bentler, P. M. (1999). Cutoff criteria for fit indexes in covariance structure analysis: conventional criteria versus new alternatives. Structural Equation Modeling, 6(1), 1-55. doi:10.1080/10705519909540118

Jackson, T., \& Chen, H. (2010). Factor structure of the Sociocultural Attitudes Towards Appearance Questionnaire-3 (SATAQ-3) among adolescent boys in China. Body Image, 7(4), 349-355. doi:10.1016/j.bodyim.2010.07.003

Kachani, A. T., Barroso, L. P., Brasiliano, S., Hochgraf, P. B., Cordás, T. A., \& Conti, M. A. (2013). Psychometric evaluation of the Body Checking Cognitions Scale (BCCS) Portuguese version. Perceptual and Motor Skills, 116(1), 175-186. doi:10.2466/08.02.15.PMS.116.1.175-186 
Kahn, J. H. (2006). Factor analysis in Counseling Psychology research, training and practice: principles, advances, and applications. The Counseling Psychologist, 24(5), 684-718. doi:10.1177/0011000006286347

Kawada, T., \& Suzuki, S. (2005). Validation study on self-reported height, weight, and blood pressure. Perceptual and Motor Skills, 101(1), 187-191.doi:10.2466/pms.101.1.187-191

Knauss, C., Paxton,S S. J., \& Alsaker, F. D. (2009). Validation of the German version of the Sociocultural Attitudes Towards Appearance Questionnaire (SATAQ-G). BodyImage, 6(2), 113-120.doi:10.1016/j.bodyim.2009.01.002

Lattin J., Carroll J. D., \& Green P. E. (2011). Análise de dados multivariados. São Paulo: Gencage Learning.

López-Guimerá, G., \& Sánchez-Carracedo, D. (2010). Prevención de lasalteraciones alimentarias: fundamentos teóricos y recursos práticos. Madrid: Pirâmide.

Madanat, H. N., Hawks, S. R., \& Brown, R. B. (2006). Validation of the Sociocultural Attitudes Towards Appearance Questionnaire-3 among a random sample of Jordanian women. Body Image, 3(4), 421-425.doi:10.1016/j.bodyim.2006.09.004

Markland, D., \& Oliver, E. J. (2008). The Sociocultural Attitudes Questionnaire-3: A confirmatory factor analysis. Body Image, 5(1), 116-121.doi:10.1016/j.bodyim.2007.10.001

Pilati, R., \& Laros, J. A. (2007). Modelos de equações estruturais em psicologia: conceitos e aplicações. Psicologia: teoria e pesquisa, 23(2), 205-216.doi:10.1590/S0102-37722007000200011

Raykov, T., \&Marcoulides, G. A. (2011). Introduction to psychometric theory. New York: Taylor \& Francis.

Rousseau, A., Valls, M., \&Chabrol, H. (2010). The Sociocultural Attitudes Towards Appearance Scale-3 (SATAQ-3): étude de validation de la version française. L'Encéphale, 36(4), 270-276. doi:10.1016/j.encep.2008.03.013

Sánchez-Carracedo, D., Barrada, J. R., López-Guimerà, G., Fauquet, J., Almenara, C.A., \& Trepat, E. (2012). Analysis of the factor structure of the Sociocultural Attitudes Towards Appearance Questionnaire (SATAQ-3) in Spanish secondary-school students through exploratory structural equation modeling. Body Image, 9(1), 163-171. doi:10.1016/j.bodyim.2011.10.002

Scagliusi, F. B., Alvarenga, M., Polacow, V. O., Cordás, T. A., Queiroz, G. K. O., Coelho, D. ... Lancha Jr, A. H. (2006). Concurrent and discriminant validity of the Stunkard's Figure Rating Scale adapted into Portuguese. Appetite, 47(1), 77-82. doi:10.1016/j.appet.2006.02.010
Stefanile, C., Matera, C., Nerini, A., \&Pisani, E. (2011). Validation of an Italian version of the Sociocultural Attitudes Towards Appearance Questionnaire-3 (SATAQ-3) on adolescent girls. Body Image, 8(4), 432-436. doi:10.1016/j.bodyim.2011.06.001

Swami, V. (2009).An examination of the factor structure of the Sociocultural Attitudes Towards Appearance Questionnaire-3 in Malaysia. BodyImage, 6(2), 129-132.doi:10.1016/j. bodyim.2009.01.003

Swami, V., Campana, A. N. N. B., Ferreira, L., Barretta, S., Harrisa, A. S., \& Tavares, M. C. G. C. F. (2011). The Acceptance of Cosmetic Surgery Scale: initial examination of its factor structure and correlates among Brazilian adults. Body Image, 8(2), 179-185.doi:10.1016/j.bodyim.2011.01.001

Tiggemann, M. (2011). Sociocultural Perspectives on Human appearance and Body Image. In T. F. Cash \& L. Smolak (Eds.), Body image: A handbook of science, practice, and prevention. (2nd ed., pp. 12-19). New York: The Guilford Press.

Thompson, B. (2004). Exploratory and confirmatory factor analysis. Washington, DC: American Psychological Association.

Thompson, J. K., Coovert, M. D., \& Stormer, S. (1999). Body image, social comparison, and eating disturbance: A covariance structure modeling investigation. International Journal of Eating Disorders, 26(1), 43-51.doi:10.1002/(SICI)1098108X(199907)26:1\%3C43::AID-EAT6\%3E3.3.CO;2-I

Thompson, J. K., van den Berg, P., Roehrig, M., Guarda, A. S., \& Heinberg, L. J. (2004). The Sociocultural Attitudes Towards Appearance Scale-3 (SATAQ-3): Development and validation. International Journal of Eating Disorders, 35(3), 293-304. doi:10.1002/eat.10257

Túry, F., Güleç, H., \&Kohls, E. (2010).Assessment methods for eating disorders and body image disorders. Journal of Psychosomatic Research, 69(6), 601-611.doi:10.1016/j. jpsychores.2009.05.012

Wertheim, E. H., \& Paxton, S. J. (2011). Body Image development in adolescent girls. In T. F. Cash \& L. Smolak (Eds.), Body image: A handbook of science, practice, and prevention. (2nd ed., pp. 76-84). New York: The Guilford Press.

Wheeler D. L., Vassar M., \& Hale W. D. (2011). A genderbased measurement invariance study of the Sociocultural Attitudes Towards Appearance Questionnaire-3. Body Image, 8, 168-172. http://dx.doi.org/10.1016/j.bodyim.2010.12.004 